\title{
Angiographic characteristics of contralateral asymptomatic eyes in acute Central serous chorio-retinopathy in an Asian population
}

\author{
Rajya L Gurung ${ }^{1}$, Afaque Anwar ${ }^{2}$ \\ ${ }^{1}$ Consultant, Vitreo-retina, Biratnagar Eye Hospital, Biratnagar, Nepal, ${ }^{2}$ Statistician, MPH, Health Educator, Biratnagar \\ Eye Hospital, Biratnagar, Nepal
}

Background: Acute Central serous chorioretinopathy (CSCR) is characterised by an idiopathic circumscribed serous retinal detachment, usually confined to the central macula. Aims and Objective: We aimed to analyse the angiographic characteristics of contralateral asymptomatic eyes of patients presenting with acute CSCR in an Asian population. Materials and Methods: This is a retrospective study of 70 consecutive patients presenting with acute CSCR who underwent detailed fundus evaluation and fluorescein angiography within one year period. Results: Fluorescein angiograms of 70 patients were analysed. Male to female ratio was 10.66: 1 . The age range of patients was (24-54) years, median 37 years and mean age 38.37 years with standard deviation 6.78 . Active tobacco use was found in $34(48.6 \%)$ eyes and systemic steroid use in $2(2.8 \%)$ eyes. Majority, 48 (34.3\%) of symptomatic and all $70(100 \%)$ of contralateral asymptomatic eyes had presenting best corrected visual acuity (BCVA) of $\geq 20 / 60$. All $70(100 \%)$ of symptomatic eyes had angiographic evidence of leakage in comparison to only $25(35.7 \%)$ of contralateral asymptomatic eyes. Multi-focal leakage was seen in $21(30 \%)$ of symptomatic eyes and $6(8 \%)$ of asymptomatic eyes, inkblot leakage pattern being the most common in seen in $57(81.4 \%)$ of symptomatic and $16(22.8 \%)$ of asymptomatic eyes. The most common site of leakage was central seen in $62(88.5 \%)$ of symptomatic eyes and $19(27.1 \%)$ of contralateral asymptomatic eyes. Conclusion: The findings in our study were comparable to other Asian studies. A considerable number of patients had angiographic evidence of CSCR in the contralateral asymptomatic eye.

Key words: Central serous chorio-retinopathy; Fluorescein angiography; Visual acuity; Asian

\section{INTRODUCTION}

Central serous chorioretinopathy (CSCR) is characterised by an idiopathic circumscribed serous retinal detachment, usually confined to the central macula, caused by leakage of fluid through the retinal pigment epithelium (RPE) as defined by fluorescein angiography (FA). ${ }^{1}$ CSCR is mostly unilateral in presentation, but in few cases can have bilateral presentation. ${ }^{2-4}$ There are very few studies analysing the fundus and angiographic characteristics in the contralateral asymptomatic eyes in patients with acute unilateral presentation. Bujarborua et al. in their analysis of
229 eyes have observed angiographic evidence of CSCR in $31.52 \%$ of contralateral asymptomatic eyes. ${ }^{5}$ Iida $\mathrm{T}$ et al. observed choroidal venous dilation $(36 \%)$ and choroidal hyper-fluorescence $(62 \%)$ by FA and Indocyanine green (ICG) angiography in contralateral asymptomatic eyes. ${ }^{6}$ There are various studies regarding the angiographic characteristics of acute CSCR in the Western population, ${ }^{7-9}$ but very few such studies on Asian eyes. ${ }^{10-14}$ Moreover, there are no studies from Asian population which have primarily analysed the angiographic characteristics in the asymptomatic fellow eye. This study aims at analysing the fluorescein angiographic features of CSCR in the 
asymptomatic fellow eyes in patients with acute unilateral symptomatic presentation.

\section{MATERIALS AND METHODS}

A series of 70 consecutive patients presenting with acute idiopathic CSCR to Biratnagar Eye Hospital between January 1, 2017 and Dec 31, 2017 was retrospectively studied. The study was approved by hospital review board and was carried out in accordance with the the declaration of Helsinki.

All patients with diagnosis of acute CSCR presenting for the very first time were included in the study.

CSCR was first diagnosed clinically using a binocular indirect ophthalmoscope and by biomicroscopic examination of the retina using a 90 diopter lens. The uncorrected and the best-corrected visual acuity were then recorded for both eyes separately. They were then subjected to a detailed FFA of both eyes. Acute idiopathic CSCR was defined as circumscribed serous detachment, usually confined to the central macula, caused by leakage of fluid through the retinal pigment epithelium (RPE) as defined by fluorescein angiography without other possible cause such as inflammation, exudation or choroidal neovascularisation, and with signs and symptoms lasting not more than 6 months. Cases with coexisting ocular or macular disease were excluded. Patients with prior history of CSCR and allergy to fluorescein were excluded from the study.

Unilateral symptomatic CSCR was defined as patients with signs and or symptoms of CSCR in one eye but who did not report any visual problems in the fellow eye.

All angiograms were retrieved from the digital database using the Topcon Retinal camera system (TRC 50 EX). Fluorescein angiogram (FA) of the symptomatic as well as fellow asymptomatic eye was analysed in regards to the number, location, shape, and topography of the leakage points.

A smokestack leakage first arises superiorly, resembling a smokestack, and then plumes out laterally as the angiogram progresses (Figure 1).

An inkblot leakage is manifested as a small dot leak that appears early and increases in size and intensity as the angiogram progresses (Figure 2).

A nonspecific leakage represents either patches of diffuse or focal granular hyper-fluorescence caused by retinal pigment epithelium (RPE) decompensation (Figure 3).

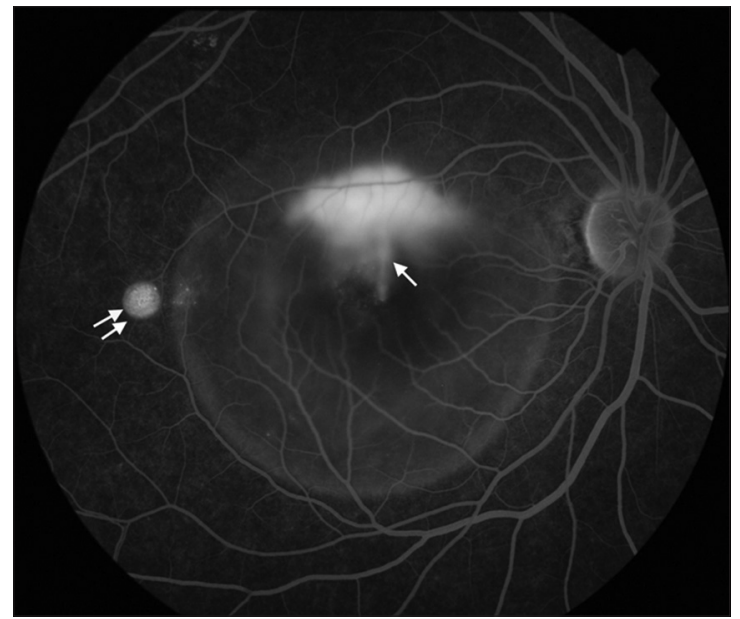

Figure 1: Single arrow showing smokestack leakage and double arrows showing PED

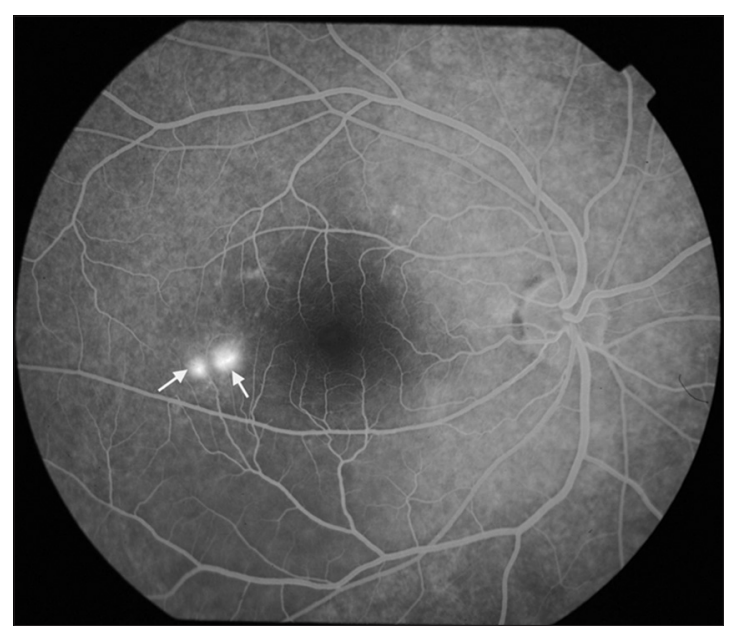

Figure 2: Multifocal inkblot leakage

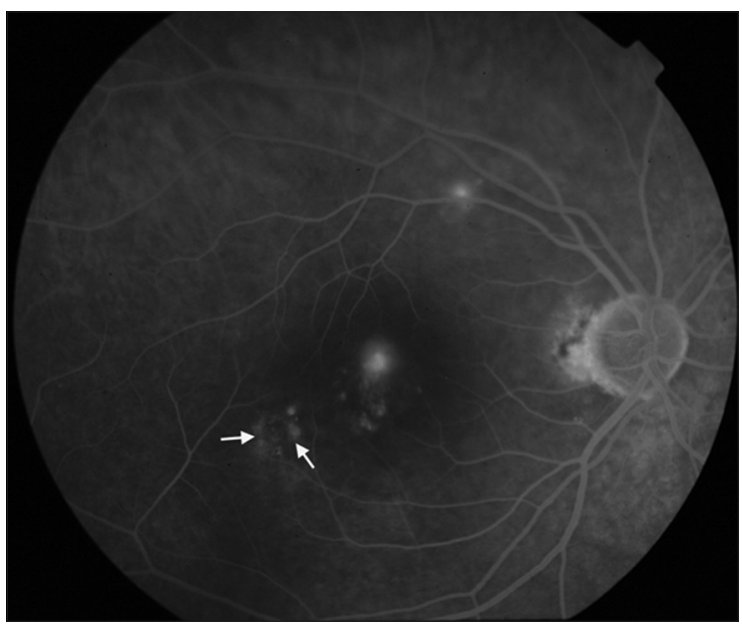

Figure 3: Arrows showing non-specific leakage

Pigment epithelial detachment (PED) revealed mostly early, and in few cases delayed, homogenous hyper-fluorescence (Figure 1). 
Mixed finding denotes any combination of above findings.

Uni-focal leakage is used to describe a solitary site of leakage while multifocal leakage is used when there is more than one site of leakage. The site of leakage will be classified into 3 groups: 1) the central 2) the peri-papillary and 3) the periphery. The central was defined as the region that coincides with the course of the major temporal blood vessels centred on the fovea with an approximate diameter of $5.5 \mathrm{~mm}$, whereas the peri-papillary area is that one within 1 disc diameter of the optic nerve, excluding the macula region, and the periphery is the area outside the macula and peri-papillary region.

Statistical Analysis: Obtained data was analysed using SPSS 16.

\section{RESULTS}

A total of 70 patients met the inclusion criteria. The majority of them were male (64/91.4\%). Male to female ratio being 10.66: 1 . There was an almost equal involvement of right eye (RE) (34/48.6\%) and left eye (LE) (36/51.4\%). The age range of patients was (24-54) years, median 37 years and mean age 38.37 years and standard deviation 6.78. Active tobacco use was found in $34(48.6 \%)$ eyes and systemic steroid use in $2(2.8 \%)$ eyes. Forty-eight $(34.3 \%)$ of symptomatic eyes had presenting best corrected visual acuity (BCVA) of $\geq 20 / 60,14(20 \%)$ had BCVA of $(<$ $20 / 60-20 / 200)$ and $8(11.4 \%)$ had BCVA $<20 / 200$. However, all $70(100 \%)$ of contralateral asymptomatic eyes had BCVA of $\geq 20 / 60$. All $70(100 \%)$ of symptomatic eyes had angiographic evidence of leakage in comparison to only $25(35.7 \%)$ of contralateral asymptomatic eyes. Among the symptomatic eyes, 49 (70\%) had single leak, $21(30 \%)$ had multiple leaks in fluorescein angiography. In the asymptomatic eyes, 19(27.1\%) had single leak and $6(8 \%)$ had multiple leaks. Inkblot was the most common type of leakage seen in $57(81.4 \%)$ of symptomatic eyes, followed by mushroom pattern seen in $5(7.1 \%)$ of eyes. Even in contralateral asymptomatic eyes, inkblot was the more common type of leakage, seen in $16(22.8 \%)$ eyes, followed by mushroom pattern in $1(1.4 \%)$ eyes, mixed pattern in $1(1.4 \%)$ eyes and non-specific leakage in $7(10 \%)$ eyes. The most common site of leakage was central seen in $62(88.5 \%)$ of symptomatic eyes and $19(27.1 \%)$ of contralateral asymptomatic eyes. Rest of the symptomatic eyes had peri-papillary leakage in $4(5.7 \%)$ eyes and remaining $4(5.7 \%)$ had leakage in mixed sites, whereas the remaining of the asymptomatic eyes had peri-papillary leakage in $4(5.7 \%)$ eyes and peripheral leakage in $2(2.8 \%)$ eyes. Very few patients had CSCR associated with PED, seen in only $5(7.1 \%)$ of symptomatic eyes and $1(1.4 \%)$ of contralateral asymptomatic eyes.

\section{DISCUSSION}

This prospective study looked at the angiographic characteristics of contralateral asymptomatic eyes of patients presenting with acute unilateral symptomatic CSCR in Asian population. The unique feature of this study is the angiographic features of acute CSCR in the contralateral asymptomatic eye in an entirely new population. Only few studies from Asia have described the angiographic features of acute CSCR. ${ }^{10-14} \mathrm{Few}$ other studies from Taiwan and China have described the epidemiology, demographics and prevalence of CSCR in Asian population. ${ }^{15,16}$

The patient demographics of acute CSCR in our population were compared to that reported in other Asian studies. The male-to-female ratio ranged from 3.9: 1 to $5.7: 1$ in Asian population, ${ }^{11-13}$ higher than reported in European and American populations. ${ }^{8}{ }^{817}$ In the present study it was even higher (10.66:1) which was similar to the finding in an Egyptian population as quoted by MM Shahin ${ }^{9}$ and in a recent study conducted in Indian population. ${ }^{10}$ As reported by MM Shahin et al. the higher male incidence might be due to greater stress in male population. The mean age of presentation of $(38.37+6.78)$ years in this study group was similar to mean age of 34.30 years and 41 years reported in earlier Asian populations. ${ }^{10,12}$

The inkblot pattern of leakage was by far the most common leakage pattern seen on angiography both in symptomatic $57(81.4 \%)$ and contralateral asymptomatic eyes $(16 / 22.8 \%)$. This was comparable to the findings in other Asian and Western studies. ${ }^{8,10,12}$ Mushroom pattern of leakage was seen in only $5(7.1 \%)$ of symptomatic eyes and $1(1.4 \%)$ of asymptomatic eyes which was far less in comparison to other Indian studies $22.51 \%{ }^{10}$ and $14.4 \% .{ }^{18}$

In this study, 25(35.7\%) of contralateral asymptomatic eyes had angiographic evidence of leakage at presentation, thus bilateral disease was seen in $35.7 \%$ which is slightly lower than reported in a Singaporean study 42\% (54/128). ${ }^{12}$ Study from Pakistan had similar finding as in our study $22(34.4 \%) \cdot{ }^{13}$ Our finding is much higher than a recent study from India. ${ }^{10}$ Only $38(10.11 \%)$ of patients had bilateral CSCR at initial visit, however the number increased to $128(34.04 \%)$ in subsequent visits. The tendency for bilaterality was found to increase with a longer follow-up in other studies also. ${ }^{2,19,20}$

Multifocal leakage was seen in 21(30\%) of symptomatic and $6(8 \%)$ of asymptomatic eyes, slightly lower than reported in 
other Asian studies (44\%-55.72\%), ${ }^{12,10}$ however exceeding those reported in non-Asian populations. ${ }^{21,22}$

Very few patients had CSCR associated with PED, seen in only $5(7.1 \%)$ of symptomatic eyes and $1(1.4 \%)$ of contralateral asymptomatic eyes similar to the finding of Perkis et al. $(8 \%) .{ }^{23}$ However, another the study done in Pakistan reported a very high incidence of PED (45.3\%). ${ }^{13}$

In the study done by Bujarborua et al. PED was exclusively found in $17.14 \%$ of the asymptomatic eyes much higher than that reported in our study. The difference might be due to the fact that our study was conducted only in acute CSCR cases and PED has been shown to be more common in chronic CSCR ${ }^{13}$

The most common site of leakage was central seen in $62(88.5 \%)$ of symptomatic eyes and $19(27.1 \%)$ of contralateral asymptomatic eyes. A similar high incidence of macular involvement has been reflected both from Asia $^{10,12}$ as well as from the non-Asian population. ${ }^{?}$

Regarding risk factor, active tobacco use in the form of Paan, ghutka, cigaratte, bidi was found to be the most common risk factor seen in $34(48.6 \%)$ eyes, this finding was much higher than reported in other studies $(9.37 \%) .{ }^{13}$ Systemic steroid use was seen in only $2(2.8 \%)$ eyes. We did not assess the personality type and the level of depression/ stress level as done in other studies. ${ }^{16,17}$ No association was found with systemic hypertension, sleep disorders or medicinal herbs.

The present study found that all $(70 / 100 \%)$ the patients had visual acuity $20 / 60$ or better in the asymptomatic eye highlighting the fact that good visual acuity might be one of the main reasons for asymptomatic presentation despite having an angiographic evidence suggestive of CSCR. Bujarborua et al.in their study also demonstrated visual acuity $20 / 30$ or better in the asymptomatic eye. ${ }^{5} \mathrm{On}$ the contrary, only $(48 / 34.3 \%)$ of patients had visual acuity better than 20/60 in the affected eye in our study.

\section{Limitations of the study:}

There were several limitations to our study. Our study involved only patients with acute CSCR, they could not be followed up and only the initial data was available. Hence the angiographic characteristics may not be representative of the entire disease spectrum, the percentage of bilaterality might have increased with follow up data. In our study, ICG angiography could not be done since it is not available in our setup. The small sample size was another limitation. To the best of our knowledge, this was the first work of its kind in Asian population. Bilateral and multifocal involvement is a common presentation. The results of this retrospective series will provide a useful basis for a more comprehensive prospective review in future.

\section{CONCLUSION}

The main outcome of this study is the categorisation of FA findings in the fellow asymptomatic eyes of cases with acute CSCR in one eye at the initial visit. This study specifically analysed the various types of findings in affected and contralateral asymptomatic eye and found that these eyes are at risk for symptomatic episodes in near future. Moreover, this study indirectly supports the view that CSCR is basically an asymmetrically developed bilateral disease process.

\section{REFERENCES}

1. Catherine B Meyerle and Richard F Spaide. Central Serous Chorioretinopathy. Albert \& Jakobiec's Principles \& Practice of Ophthalmology, Saunders 2008; 3: pp 1871-1880.

2. Yannuzzi LA, Schatz $H$ and Gitter KA. Central Serous Chorioretinopathy. The Macula: A Comprehensive Text and Atlas. Baltimore: Williams \& Wilkins; 1979; pp145-165.

3. How ACSW and Koh AH. Angiographic characteristics of acute central serous chorioretinopathy in an Asian population. Annals -Academy of Medicine Singapore 2014; 35(2):77.

4. Liew G, Quin G, Gillies M and Fraser-Bell S. Central serous chorioretinopathy: a review of epidemiology and pathophysiology. Clin Exp Ophthalmol 2013; 41:201-214.

5. Bujarborua D, Chatterjee S, Choudhury A, Bori G and Sarma AK. Fluorescein angiographic features of asymptomatic eyes in central serous chorioretinopathy. Retina 2005; 25(4): 422-429.

6. lida T, Kishi S, Hagimura $\mathrm{N}$ and Shimizu K. Persistent and bilateral choroidal vascular abnormalities in central serous chorioretinopathy. Retina (Philadelphia, Pa.)1999; 19(6): 508-512.

7. Hussain N, Baskar A, Ram LM and Das T. Optical coherence tomographic pattern of fluorescein angiographic leakage site in acute central serous chorioretinopathy. Clin Exp Ophthalmol 2006; 34:137-140.

8. Turchetti R, de Moraes HV and Maia HS. Number, shape, and topography of leakage points in patients with central serous chorioretinopathy. Arq Bras Oftalmol 2005; 68:317-320.

9. Shahin MM. Angiographic characteristics of central serous chorioretinopathy in an Egyptian population. International Journal of Ophthalmology 2013; 6(3): 342.

10. Mishra A, Baranwal VK, Aggarwal S, Shankar S, Parihar JS and Ahluwalia TS. The fluorescein angiographic characteristics of acute central serous chorioretinopathy among Indians vis-avis the other Asian and Western populations. Journal of Clinical Ophthalmology and Research 2018; 6(1): 20.

11. Eom Y, Oh J, Kim SW and Huh K. Systemic factors associated with central serous chorioretinopathy in Koreans. Korean Journal of Ophthalmology 2012; 26(4): 260-264.

12. How $\mathrm{AC}$ and $\mathrm{Koh} \mathrm{AH}$. Angiographic characteristics of acute central serous chorioretinopathy in an Asian population. Ann Acad Med Singapore 2006; 35:77-79.

13. Jamil AZ, Mirza KA, Qazi ZU, Iqbal W, Khaliq J, Fawad-urRahman, et al. Features of central serous chorioretinopathy presenting at a tertiary care hospital in Lahore. J Pak Med Assoc 
2013; 63:478-482.

14. Miura M, Elsner AE, Weber A, Cheney MC, Osako M, Usui M, et al. Imaging polarimetry in central serous chorioretinopathy. Am J Ophthalmol 2005; 140:1014-1019.

15. Tsai DC, Chen SJ, Huang CC, Chou P, Chung CM, Huang PH, et al. Epidemiology of idiopathic central serous chorioretinopathy in Taiwan, 2001-2006: a population-based study. PLoS One2013; 8(6): p.e66858.

16. Li Y, You QS, Wei WB, Xu J, Chen CX, Wang YX, et al. Prevalence and associations of central serous chorioretinopathy in elderly Chinese. The Beijing Eye Study 2011. Acta ophthalmologica 2011; 94(4): 386-390.

17. Tittl MK, Spaide RF, Wong D, Pilotto E, Yannuzzi LA, Fisher YL, et al. Systemic findings associated with central serous chorioretinopathy. American Journal of Ophthalmology 1999; 128(1):63-68.

18. Bujarborua D, Nagpal PN and Deka M. Smokestack leak in central serous chorioretinopathy. Graefes Arch Clin Exp Ophthalmol 2010;248:339-351.

19. Castro-Correia J, Coutinho MF, Rosas V and Maia J. Long-term follow-up of central serous chorioretinopathy in 150 patients. Doc Ophthalmologica 1992; 81:379-386.

20. Yap EY and Robertson DM. The long-term outcome of central serous chorioretinopathy. Arch Ophthalmol 1996; 114:689-692.

21. Spitznas $M$ and Huke J. Numbers, shape and topography of leakage points in acute type I central serous retinopathy. Graefe's Arch Clin Exp Ophthalmol 1987; 225:437-440.

22. Mutlak JA and Dutton GN. Fluorescein angiographic features of acute central serous retinopathy: A retrospective study. Acta Ophthalmol 1989; 67:467-469.

23. Perkis SL, Kim JE, Pollack JS and Merrill PT. Clinical characteristics of central serous chorioretinopathy in women. Ophthalmology 2002; 109: 262-266.

Authors Contribution:

RLG- Concept and design of the study, data collection, manuscript preparation, statistically analyzed and interpreted, critical revision of the manuscript; AA-statistical analysis.

Work attributed to: Department of Vitreo-Retina, Biratnagar Eye Hospital.

Orcid ID:

Dr. Rajya L Gurung - (1) https://orcid.org/0000-0003-0816-9823

Mr. Afaque Anwar - io https://orcid.org/0000-0002-7088-6615

Source of Support: Nil, Conflict of Interest: None. 